\title{
Limiting the Use of Verbal Coding in the Visual Patterns Test
}

\author{
Louise A. Brown, Douglas Forbes, and Jean McConnell
}

Glasgow Caledonian University

To cite:

Brown, L.A., Forbes, D., \& McConnell, J. (2006). Limiting the use of verbal coding in the Visual Patterns Test. The Quarterly Journal of Experimental Psychology, 59, 1169-1176. DOI: $10.1080 / 17470210600665954$

\section{ACKNOWLEDGEMENTS}

The authors wish to thank Dr Gerry Quinn and two anonymous reviewers for their helpful comments on earlier versions of this paper. 


\section{ABSTRACT}

The aims of the study were to assess the availability of verbal coding and its effect on performance in a standard visual matrix task, the Visual Patterns Test (VPT). In a Pilot Study, participants were presented with the patterns from the VPT, and were asked to name the shapes within them. Availability of verbal codes was low overall, however some patterns resulted in a higher mean number of labels than others. A modified version of the Test was created from those patterns which had produced the lowest mean number of labels. Sixty participants then took part in an experimental study which was carried out to assess whether or not the availability of verbal coding affects task performance. It was found that the modified version resulted in a lower visual working memory span than that of another version in which the availability of verbal coding was higher. The study confirmed that verbal coding does influence visual matrix task performance, however the modified version now offers a selection of patterns from the VPT where verbal coding has been limited.

Keywords: Visual Patterns Test; matrix pattern; verbal coding; visual memory; working memory. 
Visual matrix patterns have been used to measure visual short-term memory (STM) performance since their development by Phillips and colleagues in the 1970s (e.g. Phillips \& Baddeley, 1971). They are still being used today (e.g. Andrade, Kemps, Werniers, May, \& Szmalec, 2002; Avons \& Sestieri, 2005; Bruyer \& Scailquin, 1999; Della Sala, Gray, Baddeley, Allamano \& Wilson, 1999; Logie \& Pearson, 1997) as the research into the operation of visuo-spatial working memory has seen an increase. Given the growth in research activity in this area, it is pertinent that tasks are available which tap the intended function as exclusively as possible.

Perhaps the appeal of matrix patterns lies in the fact that they provide a simple yet flexible tool for assessing the ability simultaneously to process and store visual material. The simple cell-by-cell matrix structure is also relatively controllable and adaptable for the purposes of experimental research, in that the amount of information to be remembered can be manipulated easily by altering the number of cells. The Visual Patterns Test (VPT; Della Sala, Gray, Baddeley, \& Wilson, 1997) is a standardised test in which the stimuli are black and white square matrix patterns of increasing size which are displayed for 3 seconds and then recalled either immediately or after a short delay. The VPT has been described by Cornoldi and Vecchi (2003) as an appropriate test for measuring visuo-spatial STM. Its creators claim further that, since the Test is limited in spatio-sequential properties, it is a relatively pure measure of the visual component and that, more importantly, it is difficult to code verbally (Della Sala et al., 1997; Della Sala et al., 1999).

Phillips and Christie (1977) used black and white square matrices of a standard size in a serial position paradigm in an attempt to define the processes involved in visual STM. In doing so, they claimed that a previous but similar attempt by Cohen (1972) involved sequences of pictures which could easily be described verbally and that, as such, recall of this visual material was in fact verbal in nature. Phillips and Christie described their matrix 
patterns as abstract images for which a verbal code was not readily available. This claim was supported by Wilson, Scott, and Power (1987) who used similar stimuli which varied in size to assess visual STM span in children as well as adults. In the effort to remove the verbal coding strategy from visual memory tasks, the development of abstract patterns was desirable. However, evidence has suggested that completely excluding verbal processing resources from a visual task could be impossible.

\section{PILOT STUDY}

Paivio's (1971) dual-coding theory states that non-verbal stimuli are processed by a coding system which employs both imagery and verbal resources. These resources are seen to be independent in that their relative availability and activation can vary with the attributes of a stimulus, however they are also interconnected since the activation of one system can evoke activation of the other (Paivio, 1991). Importantly, the theory holds that images, both concrete and abstract, can be translated into words. If the image is unfamiliar or abstract, as matrix patterns are said to be, then the availability of verbal coding should indeed be lower. Crucially, however, verbal coding would still be available to some degree.

In relation to the VPT, the fact that the stimuli are in the form of abstract patterns and not, for example, images of everyday objects, means that the availability of verbal coding should be relatively limited. However, as Avons and Phillips (1987) noted, matrix patterns may be comprised of both unfamiliar and familiar shapes. While a given pattern would be classified as abstract when the overall configuration is taken into account, it is possible that the random, familiar shapes present within some of the patterns could be deemed as concrete and therefore more amenable to verbal coding. This issue was addressed in a recent study which involved the use of the VPT. Cocchini, Logie, Della Sala, MacPherson, and Baddeley (2002: p1088) carried out a study which investigated the separability of verbal and non- 
verbal memory and, in using the VPT to assess non-verbal memory, chose to exclude "patterns that obviously formed canonical shapes such as letters or numbers". No detail was provided with regards to which or how many patterns were excluded, however it would seem that the basis upon which the patterns were excluded was subjective. This study has acknowledged that some patterns consist of shapes which can be verbally coded and, given the importance of matrix tasks in the study of visual STM, it is essential that verbal coding in the VPT is examined more objectively.

First, it is questionable whether only canonical, familiar shapes are obvious in some of the patterns. More elaborate constructs may also be obvious to some individuals. Second, it is important to question whether the degree of verbalisability is uniform across the stimuli, both within and across the levels of complexity. If some of the patterns were found to be more amenable to verbal coding than others, the current study aimed to create a modified version of the VPT which limits verbal coding and provides a more specific tool for the measurement of visual STM.

\section{Method}

\section{Design}

The effects of pattern number and level of complexity were investigated on two dependent variables. The first dependent variable was the number of labels provided. The second dependent variable was the participants' self-ratings of the amount of each pattern that had been coded verbally.

\section{Participants}

The participants were 28 volunteers who were either known to the experimenter or were students at the University. The sample comprised 18 females and 10 males. The mean 
age was 24.5 years $(S D=5.92$, range $=18-39)$, while the mean number of years of education was $15.7(S D=2.55$, range $=13-21)$.

\section{Materials}

The Visual Patterns Test stimulus cards, from both versions A and B, were used to display the patterns. Response sheets containing 21 five-point self-rating scales were used to allow participants to indicate the amount of each pattern that they felt they had coded verbally $($ one $=$ hardly any, five $=$ most of the pattern $)$. A dictaphone was also required to record the labels provided by the participants.

\section{Procedure}

Due to the large number of patterns which comprise versions A and B of the Test (84), it was necessary to divide the total number of patterns into smaller subgroups. Twenty-one was deemed an appropriate number of patterns to administer to any one participant, and this resulted in four groups of 21 patterns. Each group comprised a random selection of differently sized patterns from versions A and B. Although each pattern remained in the same group, administration order was random.

Participants were randomly assigned to the task of coding verbally one of the four groups of patterns. A standard instruction sheet was used to inform participants that they were going to be shown a series of black and white chequered patterns of various sizes, and that their task was to provide names or labels to describe the shapes within each pattern. Participants were also informed that the shapes may resemble "everyday objects or symbols", however the words letters and numbers were not explicitly mentioned. It was also highlighted that some patterns might be difficult to label at all. After attempting to label each pattern, participants estimated on a scale from one (hardly any) to five (most of the pattern), the 
overall quantity of each pattern which they felt they had labelled. A practice pattern was administered to ensure that the participants had fully understood the task.

Each pattern was then administered in random order. The stimulus card was displayed and participants were given unlimited time to code verbally the shapes being viewed. The comments were tape recorded. After verbal coding of each pattern, self-ratings of the degree of verbalisation were provided. This procedure continued until all 21 patterns had been administered. Participants were debriefed as regards the purpose of the study, and given the opportunity to ask questions. The time taken to complete the procedure was about 15 minutes.

Once the data had been collected, transcription and coding took place. Participants' comments for each pattern were coded into a number which the experimenter believed to be representative of the number of labels that had been provided. The experimenter adopted certain rules to facilitate objectivity. In order to focus upon the labelling of shapes that were constructed by a number of cells, those labels which coded only one cell (such as square or dot) were ignored. All labels included in the analysis therefore coded two or more individual cells. Occasionally, some labels were encountered that the experimenter did not accept to be true verbal codes. These included labels such as crossword or rubix cube, since such labels did not serve to describe any particular configuration other than a random collection of differently coloured squares. Vague labels such as office plan, or road map were deemed worthy of one label, because such labels alluded to at least one particular shape (e.g. a corridor or road represented by a rectangle). Lastly, if a label such as letter $L$ was provided, say, twice for a given pattern, this was coded as two individual labels providing they referred to two distinct shapes within the pattern. All labels, even the more elaborate ones such as giraffe, were coded as one label providing they referred to one overall configuration. 


\section{Results}

The mean number of labels was taken to be indicative of the degree of verbalisation of the patterns. The mean number of labels provided by the participants for a group of 21 patterns was $32.00(S D=16.70)$. For any one pattern, the mean number of labels was 1.50 $(S D=0.79)$ while the mean self-rating of the degree of verbal coding of any one pattern was $2.83(S D=0.87)$. A correlation analysis assessed the relationship between the mean number of labels provided and the mean self-ratings of the degree of verbalisation. The Pearson product-moment correlation coefficent revealed that these two variables were positively correlated $(r=0.47, p<.05)$.

Canonical, more basic shapes comprised a large portion of the labels that were provided, with labels such as the letter $L$, the letter $C$, rectangle, and diamond amongst them. Interestingly though, many of the labels took the form of more elaborate configurations such as animal, steps, face, and arrowhead. The mean number of canonical labels provided by participants for a group of 21 patterns was $15.54(S D=15.38)$ while that of elaborate labels was $16.04(S D=8.99)$. A paired samples t-test confirmed that the occurrence of each label type was the same, $t(27)=-.14, p=$ n.s.

Each participant's data were collapsed together to discover their mean number of labels for small (levels $2 \& 3$ ), medium (levels $8 \&$ 9), and large (levels $14 \& 15$ ) levels of complexity. As pattern size increased, so too did the mean number of labels. Small $(M=0.97$, $S D=0.70)$, medium $(M=1.60, S D=1.14)$, and large $(M=1.83, S D=1.10)$ levels of complexity were entered into a one-way repeated measures analysis of variance which was found to be highly significant, $F(2,54)=14.23, M S E=0.39, p<.001$. Paired samples t-tests identified that the mean number of labels provided for small patterns was lower than that of medium patterns, $t(27)=-3.88, p<.01$ (two-tailed), and also of large patterns, $t(27)=-5.06$, 
$p<.01$ (two-tailed). However, there was no difference between the mean number of labels provided for medium and large patterns $(t(27)=-1.38, p=n . s)$.

Appendix A lists the patterns which were selected for the modified version of the VPT as well as their mean number of labels and standard deviations. From the six available patterns existing at each level of the standard versions A and B, these were the three patterns which achieved the lowest mean number of labels. Patterns are listed (and should be administered as) the $A$ patterns in numerical order followed by the $B$ patterns in numerical order. This ensures that the patterns are administered in no particular order of the availability of verbal coding. Where two or more patterns competed for selection in a given level of complexity due to having achieved the same mean number of labels, the selection was made on the basis of the maximum number of labels or total number of labels. Most importantly, however, any patterns which were clearly relatively high in their availability of verbal coding were distinguishable from the rest and resulted in subsequent exclusion from the modified version. These remaining patterns are listed in Appendix B.

\section{Discussion}

The relatively low mean number of labels suggests that, while verbal coding is in fact possible for some of the shapes within some of the patterns, in most cases only one or two shapes could be coded verbally. The mean self-rating of the degree of the pattern that was coded verbally supports the claim that, although verbalisation occurred, it tended to be for only some of the shapes within the patterns and not for all of the information contained therein. This evidence supports the validity of the VPT as a visual task because, since the Test requires patterns to be recalled completely correctly, participants must rely upon their memory for the whole visual image in order to progress. The study has therefore supported previous claims that matrix patterns are difficult to code verbally (Della Sala et al., 1997; 
Della Sala et al., 1999; Phillips \& Christie, 1977; Wilson et al., 1987). However, the results are interesting in that they do clearly show some degree of verbal coding. The study also confirmed that, where participants provided a higher number of labels, they also believed that a greater portion of the pattern had been coded verbally.

Two particularly interesting results emerged from the study. First, although the small patterns produced a lower mean number of labels than the medium and the large patterns, the large patterns did not result in significantly more labels than the medium patterns. Due to the basic cell structure of the patterns, this is likely to indicate that as their size increases, the shapes within the patterns become more complex and therefore more difficult to name. This evidence speaks to the literature on picture naming, in which the effect of stimulus complexity has received little attention (Johnson, Paivio, \& Clark, 1996). Second, while previous authors have cautioned that the patterns might contain familiar canonical shapes (Cocchini et al., 2002), the present results have shown that more elaborate shapes can also be identified. In fact, the number of elaborate shapes was comparable with the number of canonical ones. This conflicts with the idea that only canonical shapes and symbols can be formed from the square matrix design of the stimuli.

While the Pilot Study has provided further information regarding the availability of verbal coding in the VPT, it remains to be seen whether this factor influences task performance. Experiment 1 was designed to investigate this issue by comparing memory for the patterns that were included in the modified version of the Test with those that were excluded.

\section{EXPERIMENTAL STUDY}

In order to determine what functions are measured by the VPT, Experiment 1 sought to determine whether the availability of verbal coding affects task performance. Paivio (1991) 
provided evidence that participants display superior recall for pictures of concrete objects compared with the words of concrete objects, and also that recall of concrete words is superior to recall of abstract words. Over many years the concreteness effect has been shown to be robust and is also supported by recent evidence including that of Richardson (2003). This suggests that the utilisation of both processing systems exerts an additive effect upon subsequent memory performance due to the increased size of the available processing resources. Although the theory holds that abstract pictures will result in a lower degree of verbal coding than concrete pictures, it remains possible that even a small degree of verbal coding could result in increased pattern recall in the VPT. It could be argued that those VPT patterns which are lower in their availability of verbal coding are more abstract than those patterns which are higher in their availability of verbal coding.

However, the possibility of participants adopting a verbal coding strategy in matrix pattern tasks has been investigated before by Pickering, Gathercole, Hall, and Lloyd (2001). They compared the immediate recall of matrix patterns by children and adults under control conditions with that of an articulatory suppression condition in which the ability to process the information verbally was limited. The results showed that articulatory suppression did not reduce task performance, suggesting that participants do not rely on a verbal coding strategy, but a visuo-spatial one for matrix task completion. It is possible that the procedure adopted by Pickering and colleagues was not amenable to a verbal coding strategy. With the current Pilot Study being designed to determine the availability of verbal coding, its procedure allowed participants unlimited time to study the patterns and assign verbal labels. It is therefore important to consider that the availability of verbal coding may not be the same when the time constraints of the standard procedure are taken into account. Based on the evidence of Pickering et al. (2001) and on the fact that the verbalisation of the VPT patterns provided by participants in the Pilot Study was performed under no time-constraint, it was expected that 
participants would adopt a visual coding strategy for task completion. On this basis, it was expected that no reliable difference in performance would be found between conditions of low and high verbalisability.

\section{Method}

\section{Participants}

Participants were 60 staff and students of the University. Their mean age was 28.85 years $(S D=5.53$, range $=20-40)$ and the mean number of years of education was $18.00(S D$ $=2.99$, range $=11-25)$. Thirty-six of the participants were female and 24 were male. Each participant was paid $£ 3$ for their time. Immediately prior to being tested, participants had taken part in a short, separate test of spatial memory, lasting around 15 minutes. The current experiment therefore controlled for the previous experimental condition to which each participant had belonged.

\section{Design}

The experiment took the form of a between-subjects design, which assessed the effect of the availability of verbal coding upon visual working memory span. Availability of verbal coding was either low or high.

\section{Materials}

The stimuli were the VPT patterns that were either low (see Appendix A) or high (see Appendix B) in their availability of verbal coding, as determined by the Pilot Study. The stimuli were presented on a laptop monitor and recall of the patterns took place on blank paper templates. 


\section{Procedure}

Participants were asked to read a standard set of instructions before carrying out a practice trial. After the practice, the Test began at the fourth level of complexity (the same level as the practice pattern). Each trial began with the participants pressing the space bar. A centred fixation cross was then viewed for a period of 2 seconds, after which the to-beremembered pattern was displayed for 3 seconds. After the pattern disappeared, the screen turned blank (white). A delay period of 10 seconds was interposed between stimulus presentation and recall in order to place greater demands on the operation of working memory. The participant was specifically instructed to continue to look at the screen while trying to concentrate on remembering the pattern. After the delay period had passed, the word recall appeared on the centre of the screen. The participant then recalled the pattern on the paper templates by placing crosses in the cells that they remembered as being black. The experimenter provided feedback regarding whether or not recall had been successful. The procedure continued until the participant failed to recall correctly at least one of the patterns from a given level of complexity. Visual working memory span was taken to be the mean size of the last three correctly recalled patterns.

\section{Results}

The mean score resulting from low verbalisability was $8.72(S D=1.55)$, while that of high verbalisability was $10.08(S D=2.09)$. In order to determine whether or not this difference was reliable, an independent samples t-test was carried out. The analysis confirmed that performance was better when the availability of verbal coding was higher $(t(58)=-2.86, p<$ .01 , two-tailed). 


\section{Discussion}

Given that the one systematic difference between the two sets of stimuli was the availability of verbal coding, the results indicate first that the participants adopted a dualcoding strategy where available. Second, the increased availability of verbal coding enabled participants to remember more visual information. These results support Paivio's (1971; 1991) dual-coding theory in terms of the increased memory capacity related to the availability of both verbal and imaginal coding.

In terms of visual matrix tasks, contrary to the study by Pickering et al. (2001), the current experiment provides evidence that participants do utilise a verbal coding strategy where available, and that this strategy aids task performance. It is possible that the opportunity for dual-coding is reduced when the task features immediate recall, as did the study by Pickering and colleagues. This issue warrants further investigation in order to promote our understanding of the strategies available to participants under different procedural constraints. Nevertheless, the present study indicates that matrix tasks can incorporate some measurement of verbal memory performance, particularly if the stimuli have not been screened to exclude those patterns which are more amenable to verbal coding. The modified version of the VPT has currently been shown to limit verbal coding and to measure more specifically the operation of visual working memory.

\section{GENERAL DISCUSSION}

The present study has helped us to understand better the availability of verbal coding of the matrix patterns involved in the VPT, as well as the importance of this factor to task performance. The study showed that in some patterns verbal coding is more readily available than in others, however, the overall amount of labelling was small in relation to the amount of information to be remembered. With the task requiring patterns to be recalled completely 
correctly, the task is a valid measure of visual memory. This validity can be increased further with more specific measurement of visual memory performance, when adopting the use of the modified version of the VPT which limits both the availability of verbal coding and therefore the opportunity for participants to adopt a dual-coding strategy. The study extends to those researchers developing their own matrix patterns for the measurement of visual memory performance, since the procedure adopted in the pilot study was shown to serve as a useful tool for screening potential patterns for inclusion in a matrix-type task. 


\section{REFERENCES}

Andrade, J., Kemps, E., Werniers, Y., May, J., \& Szmalec, A. (2002). Insensitivity of visual short-term memory to irrelevant visual information. Quarterly Journal of Experimental Psychology, 55A, 753-774.

Avons, S. E., \& Phillips, W. A. (1987). Representation of matrix patterns in long- and shortterm visual memory. Acta Psychologica, 65, 227-246.

Avons, S. E., \& Sestieri, C. (2005). Dynamic visual noise: No interference with visual shortterm memory or the construction of visual images. European Journal of Cognitive Psychology, 17, 405-424.

Bruyer, R., \& Scailquin, J. (1999). Assessment of Visuospatial short-term memory and effect of aging. European Review of Applied Psychology, 49, 175-180.

Cocchini, G., Logie, R. H., Della Sala, S., MacPherson, S. E., \& Baddeley, A. (2002).

Concurrent performance of two memory tasks: Evidence for domain-specific working memory systems. Memory \& Cognition, 30 (7), 1086-1095.

Cohen, G. (1972). Serial position effects in the recall of picture sequences. Quarterly Journal of Experimental Psychology, 24, 41-47.

Cornoldi, C., \& Vecchi, T. (2003). Visuo-spatial working memory and Individual Differences. Hove, U.K.: Psychology Press.

Della Sala, S., Gray, C., Baddeley, A., Allamano, N., \& Wilson, L. (1999). Pattern span: A tool for unwelding visuo-spatial memory. Neuropsychologia, 37, 1189-1199.

Della Sala, S., Gray, C., Baddeley, A., \& Wilson, L. (1997). Visual Patterns Test: a test of short-term visual recall. Suffolk, England: Thames Valley Test Company.

Johnson, C. J., Paivio, A., \& Clark, J. M. (1996). Cognitive components of picture naming. Psychological Bulletin, 120, 113-139. 
Logie, R. H., \& Pearson, D. G. (1997). The inner eye and the inner scribe of visuo-spatial working memory: Evidence from developmental fractionation. European Journal of Cognitive Psychology, 9, 241-257.

Paivio, A. (1971). Imagery and verbal processes. New York: Holt, Rinehart and Winston.

Paivio, A. (1991). Images in Mind: The Evolution of a Theory. Hertfordshire: Harvester Wheatsheaf.

Phillips, W. A. \& Baddeley, A. D. (1971). Reaction time and short-term visual memory. Psychonomic Science, 22, 73-74.

Phillips, W. A., \& Christie, D. F. M. (1977). Components of visual memory. Quarterly Journal of Experimental Psychology, 29, 117-133.

Pickering, S. J., Gathercole, S. E., Hall, M., \& Lloyd, S. A. (2001). Development of memory for pattern and path: Further evidence for the fractionation of visuo-spatial memory. Quarterly Journal of Experimental Psychology, 54A, 397-420.

Richardson, J. T. E. (2003). Dual coding versus relational processing in memory for concrete and abstract words. European Journal of Cognitive Psychology, 15, 481-509.

Wilson, J. T. L., Scott, J. H., \& Power, K. G. (1987). Developmental differences in the span of visual memory for pattern. British Journal of Developmental Psychology, 5, 249255. 


\section{APPENDIX A}

Mean number of labels provided (and standard deviations) for patterns included in the modified version of the Visual Patterns Test

\begin{tabular}{|c|c|c|c|}
\hline \multirow[b]{2}{*}{ Level of complexity } & \multicolumn{3}{|c|}{ Low Verbalisability } \\
\hline & Pattern 1 & Pattern 2 & Pattern 3 \\
\hline \multirow[t]{2}{*}{ Level 2} & A3 & B1 & B2 \\
\hline & $M=0.58, S D=0.53$ & $M=1.00, S D=0.00$ & $M=0.86, S D=0.38$ \\
\hline \multirow[t]{2}{*}{ Level 3} & A6 & B4 & B5 \\
\hline & $M=0.71, S D=0.49$ & $M=0.57, S D=0.53$ & $M=0.29, S D=0.49$ \\
\hline \multirow[t]{2}{*}{ Level 4} & A8 & A9 & B7 \\
\hline & $M=0.71, S D=0.49$ & $M=1.00, S D=0.82$ & $M=0.86, S D=0.69$ \\
\hline \multirow[t]{2}{*}{ Level 5} & A11 & $\mathrm{A} 12$ & B12 \\
\hline & $M=1.00, S D=1.15$ & $M=0.86, S D=0.90$ & $M=1.14, S D=0.90$ \\
\hline \multirow[t]{2}{*}{ Level 6} & A14 & A 15 & B15 \\
\hline & $M=1.29, S D=1.12$ & $M=1.57, S D=0.98$ & $M=1.57, S D=1.27$ \\
\hline \multirow[t]{2}{*}{ Level 7} & A17 & B16 & B17 \\
\hline & $M=1.00, S D=0.58$ & $M=0.29, S D=0.49$ & $M=1.14, S D=0.90$ \\
\hline \multirow[t]{2}{*}{ Level 8} & A21 & B20 & B21 \\
\hline & $M=0.71, S D=0.76$ & $M=1.14, S D=0.90$ & $M=1.43, S D=1.27$ \\
\hline \multirow[t]{2}{*}{ Level 9} & $\mathrm{~A} 23$ & B23 & B24 \\
\hline & $M=1.14, S D=0.69$ & $M=1.14, S D=0.69$ & $M=0.57, S D=1.13$ \\
\hline \multirow[t]{2}{*}{ Level 10} & $\mathrm{~A} 25$ & B25 & B26 \\
\hline & $M=1.57, S D=1.51$ & $M=1.43, S D=0.98$ & $M=1.00, S D=0.58$ \\
\hline \multirow[t]{2}{*}{ Level 11} & A29 & B29 & B30 \\
\hline & $M=1.57, S D=0.98$ & $M=0.86, S D=1.07$ & $M=0.71, S D=0.49$ \\
\hline \multirow[t]{2}{*}{ Level 12} & A33 & B32 & B33 \\
\hline & $M=1.14, S D=0.69$ & $M=1.71, S D=1.80$ & $M=2.14, S D=1.22$ \\
\hline \multirow[t]{2}{*}{ Level 13} & A35 & A36 & B35 \\
\hline & $M=1.86, S D=1.77$ & $M=1.43, S D=2.30$ & $M=1.14, S D=1.22$ \\
\hline \multirow[t]{2}{*}{ Level 14} & A39 & B38 & B39 \\
\hline & $M=1.00, S D=0.82$ & $M=1.43, S D=1.40$ & $M=1.43, S D=1.72$ \\
\hline \multirow{2}{*}{ Level 15} & $\mathrm{~A} 41$ & B 41 & B 42 \\
\hline & $M=1.71, S D=2.06$ & $M=1.71, S D=1.11$ & $M=1.86, S D=1.22$ \\
\hline
\end{tabular}




\section{APPENDIX B}

Mean number of labels provided (and standard deviations) for patterns excluded from the modified version of the Visual Patterns Test

\begin{tabular}{|c|c|c|c|}
\hline \multirow[b]{2}{*}{ Level of complexity } & \multicolumn{3}{|c|}{ High Verbalisability } \\
\hline & Pattern 1 & Pattern 2 & Pattern 3 \\
\hline \multirow[t]{2}{*}{ Level 2} & A1 & A2 & B3 \\
\hline & $M=1.14, S D=1.07$ & $M=1.00, S D=0.58$ & $M=1.43, S D=0.79$ \\
\hline \multirow[t]{2}{*}{ Level 3} & A4 & A5 & B6 \\
\hline & $M=2.00, S D=1.00$ & $M=0.71, S D=0.76$ & $M=0.71, S D=0.76$ \\
\hline \multirow{2}{*}{ Level 4} & A7 & B8 & B9 \\
\hline & $M=1.14, S D=0.69$ & $M=1.29, S D=0.95$ & $M=1.57, S D=1.13$ \\
\hline \multirow[t]{2}{*}{ Level 5} & A10 & B10 & B11 \\
\hline & $M=1.71, S D=1.25$ & $M=1.14, S D=1.35$ & $M=1.43, S D=0.79$ \\
\hline \multirow[t]{2}{*}{ Level 6} & A13 & B13 & B14 \\
\hline & $M=2.00, S D=1.29$ & $M=1.71, S D=0.76$ & $M=3.00, S D=2.89$ \\
\hline \multirow[t]{2}{*}{ Level 7} & A16 & A18 & B18 \\
\hline & $M=1.71, S D=1.50$ & $M=1.28, S D=0.95$ & $M=1.71, S D=1.25$ \\
\hline \multirow[t]{2}{*}{ Level 8} & A19 & A20 & B19 \\
\hline & $M=2.14, S D=2.12$ & $M=2.57, S D=1.13$ & $M=1.57, S D=1.51$ \\
\hline \multirow[t]{2}{*}{ Level 9} & A22 & A24 & B22 \\
\hline & $M=2.57, S D=2.23$ & $M=1.71, S D=1.70$ & $M=2.14, S D=1.57$ \\
\hline \multirow{2}{*}{ Level 10} & A26 & A27 & B27 \\
\hline & $M=2.43, S D=2.07$ & $M=1.57, S D=1.40$ & $M=2.00, S D=1.53$ \\
\hline \multirow[t]{2}{*}{ Level 11} & A28 & A30 & B28 \\
\hline & $M=1.71, S D=1.38$ & $M=2.43, S D=2.23$ & $M=1.86, S D=2.12$ \\
\hline \multirow[t]{2}{*}{ Level 12} & A31 & A32 & B31 \\
\hline & $M=2.14, S D=1.87$ & $M=2.14, S D=1.77$ & $M=2.43, S D=2.23$ \\
\hline \multirow[t]{2}{*}{ Level 13} & A34 & B34 & B36 \\
\hline & $M=2.57, S D=1.40$ & $M=2.14, S D=1.57$ & $M=2.29, S D=1.38$ \\
\hline \multirow[t]{2}{*}{ Level 14} & A37 & A38 & B37 \\
\hline & $M=2.00, S D=2.00$ & $M=2.71, S D=0.95$ & $M=2.14, S D=1.46$ \\
\hline \multirow{2}{*}{ Level 15} & A40 & A42 & B40 \\
\hline & $M=2.00, S D=1.16$ & $M=2.00, S D=1.83$ & $M=2.00, S D=1.92$ \\
\hline
\end{tabular}

